Math. Model. Nat. Phenom.

Vol. 2, No. 4, 2007, pp. 122-134

\title{
A minimal model of pursuit-evasion in a predator-prey system
}

\author{
Y. Tyutyunov ${ }^{a 1}$, L. Titova ${ }^{a}$ and R. Arditi $^{b}$ \\ ${ }^{a}$ Laboratory of Mathematical Modelling of Biological Processes, \\ Department of Mathematical Modelling in Economics and Ecology, \\ Vorovich Research Institute of Mechanics and Applied Mathematics, \\ Southern Federal University, \\ 200/1 Stachki street, 344090 Rostov-on-Don, Russia \\ ${ }^{b}$ Écologie des populations et communautés, \\ AgroParisTech, 16 rue Claude Bernard, 75231 Paris cedex 05, France
}

\begin{abstract}
A conceptual minimal model demonstrating spatially heterogeneous wave regimes interpreted as pursuit-evasion in predator-prey system is constructed and investigated. The model is based on the earlier proposed hypothesis that taxis accelerations of prey and predators are proportional to the density gradient of another population playing a role of taxis stimulus. Considering acceleration rather than immediate velocity allows obtaining realistic solutions even while ignoring variations of total abundances of both modelled populations. Linear analysis of the model shows that stationary homogeneous regime becomes oscillatory unstable with respect to small heterogeneous perturbations if either taxis activities or total population abundances are high enough. The ability for active directed movement of both prey and predators is the necessary condition for spatial self-organization. Numerical simulations illustrate analytical results. The relation between the proposed model and conventional two-component systems with cross-diffusion is discussed.
\end{abstract}

Key words: taxis-diffusion-reaction, attraction-repulsion, prey-taxis, tropho-taxis, population clustering, population wave, spatial heterogeneity, spatial behaviour

AMS subject classification: $92 \mathrm{C} 15$

\footnotetext{
${ }^{1}$ Corresponding author. E-mail: tyutyunov@sfedu.ru
} 


\section{Introduction}

Spatial heterogeneity of population distribution is one of the most intensively studied areas of theoretical biology. This phenomenon is widely spread in the nature; actually, it would be difficult to imagine an ecological system homogeneously distributed in space.

Spatial heterogeneity is observed in different biotopes [5] and can be caused by various reasons [16]. Among these reasons are spatial gradients of environmental factors that directly influence ecosystem productivity $[42,5]$, temporal local variations of population reproduction, that are caused by periodic or irregular external forces $[8,23]$, periodic boundary conditions [46], complex interplay between local kinetics and spatial fluxes of population densities $[37,27,26,30]$, etc. Perhaps, the simplest and, from our point of view, the most interesting case is the population heterogeneity caused solely by spatial behaviour of animals. In particular, we will consider here self-organized spatial structures in predator-prey systems that emerge due to pursuit-evasion activity of interacting populations.

Pitcher and Parrish [32] classified typical behaviour patterns observed in fish populations and described several examples of spatial configurations (flash expansion, ball, avoidance, herd, cruise, split, joinder, vacuole, hourglass, etc.), caused, in particular, by spatial interactions of prey and predators (see also $[29,33,21]$ ). Such pursuit-evasion movements occur at much faster temporal scale than birth/death processes, and faster even than consumption of prey by predators because the predation efficiency (their ability to capture prey after an attack) may be quite low for some species, especially if prey exhibit active escaping behaviour reducing the risk of predation $[21,33]$. The estimations of the proportion of successful attack made for various species indicate that only few attacks performed by the predators pursuing their prey are successful $[22,17,34]$.

Building mathematical models able to reproduce such a behaviourally induced spatial structuring is a challenging theoretical problem. Correct models must exhibit heterogeneous solutions under minimal assumptions and complexity. The conception of minimal model is extremely useful because it takes into account only basic features of the phenomena being considered $[31,23]$. Besides arousing pure theoretical interest, this objective has obvious practical importance because seeking the minimal description commensurate with the complexity of the problem forces one to choose among several theories that explain a given observation, the simplest and the most general one, which thereby will be potentially more robust in practical applications $[13,12]$.

One of the most efficient approaches to modelling spatio-temporal dynamics of interacting populations, that allows combining strict formulation and analytical methods with powerful algorithms of numerical investigations is based on the use of partial differential equations of the reaction-advection-diffusion type $[25,26,19,7,10,27,30]$. Remaining in this framework, we will suggest a minimal model providing obtaining heterogeneous solutions that can be interpreted as pursuit-evasion in predator-prey system.

The balance equation for population density (of prey) can be presented in the form:

$$
\frac{\partial N}{\partial t}=f(N, P)-\operatorname{div}(\mathbf{v} \cdot N)+\delta \Delta N
$$


where $N=N(t, \mathbf{x})$ is the density of population at time $t$ at spatial position $\mathbf{x}$ of domain $\Omega$; $f(N, P)$ is the function of local kinetics (dependence of birth/death processes on intra- and inter-species interactions at spatial position $\mathbf{x}) ; \mathbf{v}=\mathbf{v}(\mathbf{x})$ is the velocity of the advective flux of population density; $\delta$ is the diffusion coefficient; $\Delta$ is the Laplacian.

The advective term $-\operatorname{div}(\mathbf{v} \cdot N)$ in the balance equation (1.1) may be interpreted not only as passive transport of animals by wind, water flow, etc., but also as active taxis.

The conventional hypothesis of modelling taxis assumes dependence of taxis velocity $\mathbf{v}$ from the gradient of some stimulus $\nabla S$; in the simplest case - their proportionality: $\mathbf{v}=\kappa \nabla S$ $[25,26,7]$. The sign of the taxis coefficient $\kappa$ is defined by the taxis kind: for the so-called positive taxis (movements of individuals upward the gradient of attracting stimulus) $\kappa>0$; in the case of negative taxis (movements of organisms downward the gradient $\nabla S$ of repulsing stimulus) $\kappa<0$.

\section{Two alternative approaches to modelling pursuit- evasion processes}

The problem of describing pursuit-evasion processes was considered by various authors. There are several models based on the reaction-advection-diffusion systems (see [26] for further references and examples). Citing from a recent instance, we would like to consider here a pursuit-evasion model investigated by Tsyganov and co-authors $[40,41,39,38]$ :

$$
\begin{aligned}
& \frac{\partial N}{\partial t}=r N(1-N)-\frac{P N^{2}}{a^{2}+N^{2}}+\kappa_{N} \operatorname{div}(N \nabla P)+\delta_{N} \Delta N, \\
& \frac{\partial P}{\partial t}=e \frac{P N^{2}}{a^{2}+N^{2}}-\mu P-\kappa_{P} \operatorname{div}(P \nabla N)+\delta_{P} \Delta P,
\end{aligned}
$$

where $N$ and $P$ are densities of prey and predator populations respectively; $\mathbf{v}_{P}=\kappa_{P} \nabla N$ is the velocity of predators pursuing preys (positive taxis); $\mathbf{v}_{N}=-\kappa_{N} \nabla P$ is the velocity of preys avoiding predators (negative taxis). Hereinafter let us assume that $\kappa_{N}, \kappa_{P}>0$.

Thus, the local kinetics of model (2.2) consists of logistic reproduction of prey, Holling type-III trophic function, and constant rate of predator mortality. The authors have demonstrated that the model has very interesting spatially heterogeneous regimes including solitonic $[40,41]$ and spiral waves [38]. Basing on the mathematical models of type (2.2) and experimental work with bacterial populations, such waves in excitable cross-diffusion systems have been identified as a new class of nonlinear waves [39].

Note, that spatially heterogeneous regimes exist even in simpler models of spatial interactions of prey and predators, in particular, in models for diffusive prey and able to active directed movement predators, i.e., $\kappa_{N}=0, \kappa_{P}>0[4,3]$, as well as in purely diffusive predator-prey models $\kappa_{N}=\kappa_{P}=0$ [37,25,26,27,31,23]. The closed domains $\Omega$, i.e., zero fluxes of population density through their borders $\partial \Omega$ are supposed in all mentioned cases:

$$
\left.\frac{\partial P}{\partial \mathbf{n}}\right|_{\partial \Omega}=\left.\frac{\partial N}{\partial \mathbf{n}}\right|_{\partial \Omega}=\left.\mathbf{v}_{P}\right|_{\partial \Omega}=\left.\mathbf{v}_{N}\right|_{\partial \Omega}=0 .
$$


However, in the pursuit-evasion model of $[40,41,39,38]$ (as well as in other models of taxis and diffusive movements of populations, that exhibit heterogeneous solutions under boundary conditions (2.3)) local kinetics (i.e., the birth/death processes of both populations) being coupled with spatial behaviour of species plays a critical role in emergence of spatial structures. Moreover, existence of stable spatially heterogeneous solutions requires essential nonlinearity of the functions describing local dynamics of system, in interplay with spatial behaviour of animals. Thus, although such models perfectly describe spatio-temporal dynamics of microbial trophic systems their complex regimes cannot explain spatial configurations of highly developed species like those described in $[32,29,33]$, caused solely by the pursuit-evasion processes, i.e., by reciprocal movements of prey and predators that change their local density while total population sizes remain constant.

In particular, regarding the pure pursuit-evasion model based on (2.2), i.e., equations (2.2) less the terms of local kinetics:

$$
\begin{aligned}
& \frac{\partial N}{\partial t}=\kappa_{N} \operatorname{div}(N \nabla P)+\delta_{N} \Delta N, \\
& \frac{\partial P}{\partial t}=-\kappa_{P} \operatorname{div}(P \nabla N)+\delta_{P} \Delta P,
\end{aligned}
$$

which is conservative in respect to spatially averaged population densities with zero-flux boundary conditions (2.3), i.e.,

$$
N_{0}=\frac{1}{|\Omega|} \int_{\Omega} N d \mathbf{x}=\text { const, } \quad P_{0}=\frac{1}{|\Omega|} \int_{\Omega} P d \mathbf{x}=\text { const }
$$

it is not difficult to show that model (2.3)-(2.4) does not exhibit stable spatially heterogeneous regimes. We consider this as a drawback of the conventional approach to modelling directed movement of animals and suggest here an alternative model for the pursuit-evasion in predator-prey system, that consists of PDE system

$$
\begin{aligned}
\frac{\partial N}{\partial t} & =-\operatorname{div}\left(N \mathbf{v}_{N}\right)+\delta_{N} \Delta N \\
\frac{\partial P}{\partial t} & =-\operatorname{div}\left(P \mathbf{v}_{P}\right)+\delta_{P} \Delta P \\
\frac{\partial \mathbf{v}_{N}}{\partial t} & =-\kappa_{N} \nabla P+\delta_{\mathbf{v}_{N}} \Delta \mathbf{v}_{N}, \\
\frac{\partial \mathbf{v}_{P}}{\partial t} & =\kappa_{P} \nabla N+\delta_{\mathbf{v}_{P}} \Delta \mathbf{v}_{P},
\end{aligned}
$$

and boundary condition (2.3). Model (2.6), (2.3) differs from the conventional approach to taxis modelling in assuming that acceleration of the directed movement of population density rather than its velocity per se, is determined by the density gradient of other population. Taking into account variations of taxis velocities we proceed from the fact that for highly developed species, acceleration may be more important characteristic of animal 
locomotion than speed [1]. Particularly, rapid acceleration is the key of success to both escape manoeuvres of prey and predator attacks $[9,21,33]$. The diffusion terms in the velocity equations represent forces equalizing velocities of neighbours due to social behaviour of animals $[11,18,21,24,29]$. Earlier we with different co-authors demonstrated that considering acceleration of animal taxis allows obtaining complex spatially heterogeneous regimes induced solely by tropho-taxis activity of predators even in very simple predator-prey models $[15,14,44,43,2,35]$. The same techniques was applied for describing clustering in isolated population caused by auto-taxis consisting of intra-specific attraction and repulsion $[36,43]$. Chakraborty and co-authors [6] studied emergence of spatially heterogeneous regimes in analogous tropho-taxis predator-prey model with various non-linear trophic functions. Here we apply the same technique to model appearance of the travelling wave regimes generated solely by pursuit-evasion behaviour of predators and prey. All the details of analytical and numerical methods coincide with those described in [43].

\section{The model properties}

Rescaling the population densities as $N=N / N_{0}, P=P / P_{0}$, and considering $\kappa_{P} N_{0}$ and $\kappa_{N} P_{0}$ as new taxis coefficients, the proposed model $(2.6),(2.3)$ can be nondimensionalized so that spatially averaged population densities (2.5) became $N_{0}=P_{0}=1$. The nondimentionalized model (2.6), (2.3) has a spatially homogeneous stationary regime

$$
N=1, \quad P=1, \quad \mathbf{v}_{N}=0, \quad \mathbf{v}_{P}=0,
$$

that coincides with stationary regime of nondimensionalized model (2.3)-(2.4). However, standard linear analysis (see, e.g., [25,26,43] for the technical details) shows that, unlike model (2.3)-(2.4), stationary regime (3.7) becomes unstable with respect to wavenumber $k$ of Fourier decomposition of small spatially heterogeneous perturbation, if the following inequality is satisfied:

$$
\kappa_{N} \kappa_{P}>\frac{k^{4}\left(\delta_{\mathbf{v}_{N}}+\delta_{\mathbf{v}_{P}}\right)\left(\delta_{P}+\delta_{\mathbf{v}_{P}}\right)\left(\delta_{P}+\delta_{\mathbf{v}_{N}}\right)\left(\delta_{N}+\delta_{\mathbf{v}_{P}}\right)\left(\delta_{N}+\delta_{\mathbf{v}_{N}}\right)\left(\delta_{N}+\delta_{P}\right)}{\left(\delta_{\mathbf{v}_{N}}+\delta_{\mathbf{v}_{P}}+\delta_{N}+\delta_{P}\right)^{2}} .
$$

The instability condition (3.8) implies that with any nonnegative values of diffusion coefficients $\delta_{N}, \delta_{P}, \delta_{\mathbf{v}_{N}}, \delta_{\mathbf{v}_{P}}$ (one of the four diffusion coefficients can be even zero), there are positive critical values of taxis coefficients, that correspond to bifurcation of oscillatory excitation of any mode. With increase of product $\kappa_{N} \kappa_{P}$, modes with wavenumbers $k=1,2,3$, $\mathrm{K}$ become excited one after another in ascending order. Note that only the first excited mode generates instability of the homogeneous regime (3.7), further excitation of other modes with growth of product $\kappa_{N} \kappa_{P}$ occurs when stationary distribution (3.7) is already unstable. However the whole of the set of excited modes determine the pattern of initial development of small heterogeneous perturbation of (3.7) [26]. The higher the spatial activity of both prey and predators (i.e., the larger product of $\kappa_{N}$ and $\kappa_{P}$ ), the greater number of modes become excited and, as confirmed by numerical simulations (see fig. 1), the higher spatial complexity 
will be in the wave regime that emerges and stabilizes after the homogeneous regime (3.7) becomes unstable. Figure 1 shows the instantaneous snapshot (left-to-right phase of the wave movement) of simulated periodic regimes for one-dimensional domain $\Omega=[0, L]$ that always can be scaled so that $L=1$.

Although system (2.6) does not take into account friction forces, their absence is not a crucial assumption. Adding friction terms (in a simplest linear form) into velocity equations reforms system (2.6) to

$$
\begin{aligned}
\frac{\partial N}{\partial t} & =-\operatorname{div}\left(N \mathbf{v}_{N}\right)+\delta_{N} \Delta N, \\
\frac{\partial P}{\partial t} & =-\operatorname{div}\left(P \mathbf{v}_{P}\right)+\delta_{P} \Delta P, \\
\frac{\partial \mathbf{v}_{N}}{\partial t} & =-\kappa_{N} \nabla P-\nu_{N} \mathbf{v}_{N}+\delta_{\mathbf{v}_{N}} \Delta \mathbf{v}_{N}, \\
\frac{\partial \mathbf{v}_{P}}{\partial t} & =\kappa_{P} \nabla N-\nu_{P} \mathbf{v}_{P}+\delta_{\mathbf{v}_{P}} \Delta \mathbf{v}_{P},
\end{aligned}
$$

and modifies the instability condition (3.8) as follows:

$\kappa_{N} \kappa_{P}>\frac{D^{+}\left(k, \delta_{N}, \delta_{P}, \delta_{\mathbf{v}_{N}}, \delta_{\mathbf{v}_{P}}, \nu_{N}, \nu_{P}\right)}{k^{6}\left(\delta_{\mathbf{v}_{N}}+\delta_{\mathbf{v}_{P}}+\delta_{N}+\delta_{P}\right)^{2}+2\left(\nu_{N}+\nu_{P}\right)\left(\delta_{\mathbf{v}_{N}}+\delta_{\mathbf{v}_{P}}+\delta_{N}+\delta_{P}\right) k^{4}+\left(\nu_{N}+\nu_{P}\right)^{2} k^{2}}$.

Here $\nu_{N}$ and $\nu_{P}$ are the friction coefficients for prey and predators respectively, and numerator $D^{+}\left(k, \delta_{N}, \delta_{P}, \delta_{\mathbf{v}_{N}}, \delta_{\mathbf{v}_{P}}, \nu_{N}, \nu_{P}\right)$ is a positive polynomial of the wavenumber, diffusion and friction coefficients, which is not given here because it has rather huge expression. However, the above general conclusion about appearance of spatially heterogeneous regimes induced by high spatial activity of both populations remains valid with the only exception that sufficiently high friction forces may change the order of excitation of modes. Example in fig. 2 computed for one-dimensional case of model (3.9), (2.3) shows that, unlike model (2.6), (2.3), gradual increase of product $\kappa_{N} \kappa_{P}$ in model (3.9), (2.3) for a given set of parameter values, produces the following order of modes excitation: $k=3,2,4,5,6,1,7, \ldots$. Thus, the heterogeneous regime that stabilises when the first mode with wavenumber $k=3$ becomes excited is characterized by higher spatial complexity than shown in fig. 1 (top) with a single excited mode with wavenumber $k=1$.

\section{Discussion}

We have proposed a minimal model demonstrating spatially heterogeneous wave regimes that can be interpreted as pursuit-evasion in predator-prey system. Even if taxis coefficients of prey and predator populations are low, increasing of population densities can always cause oscillatory instability of homogeneous solution (3.7). Inequality (3.8) (as well as (3.10)) suggests that spatial self-organization in model (2.6), (2.3) requires from the ability of both 

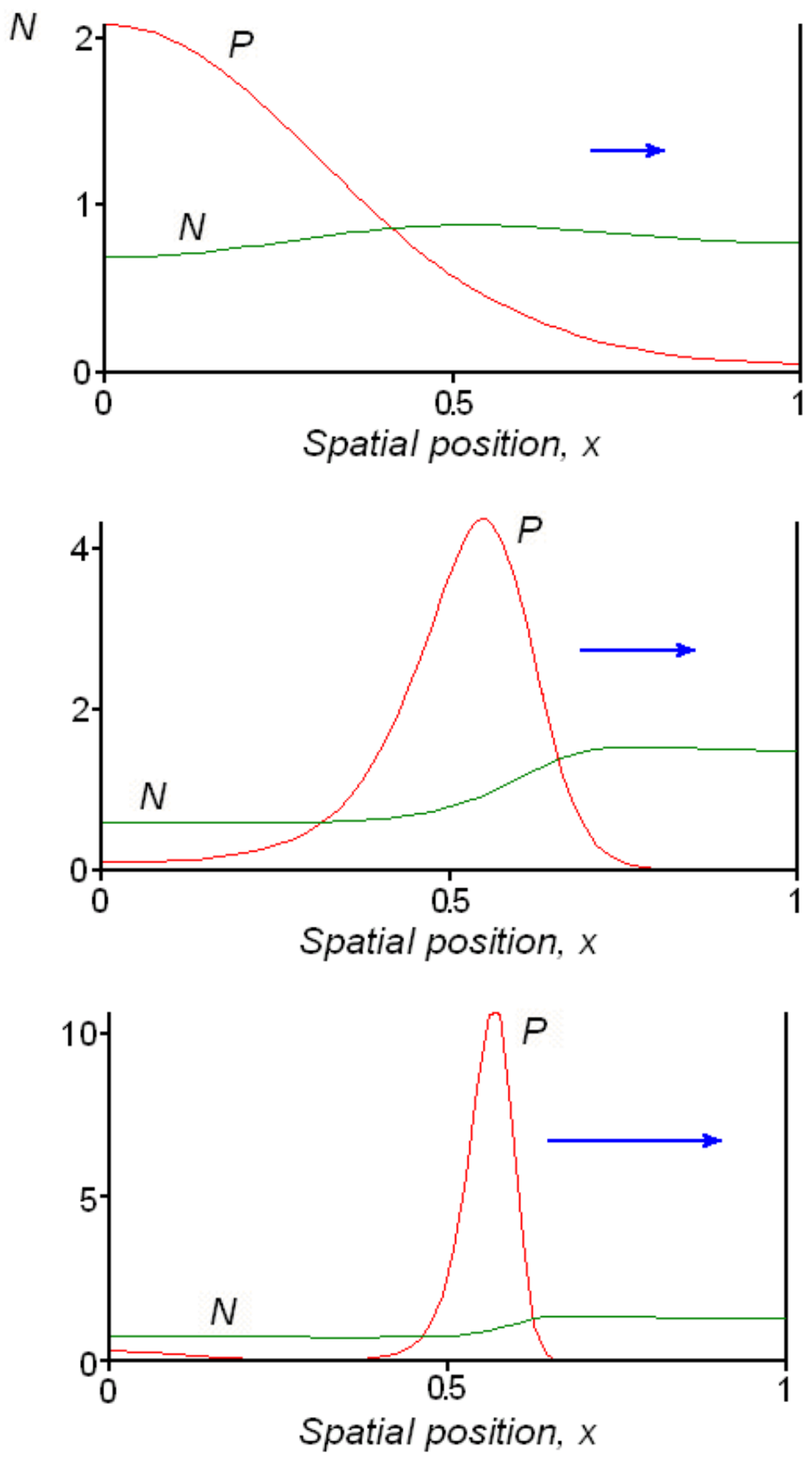

Figure 1: Time snapshots of typical spatial pattern realized in numeric simulations of travelling wave regimes in model (2.6), (2.3) with one-dimensional spatial segment $\Omega=[0,1]$. Top: one mode $k=1$ is excited, $\kappa_{N}=0.016, \kappa_{P}=0.032$; middle: two modes $k=1$ and $k=2$ are excited, $\kappa_{N}=0.02, \kappa_{P}=0.48$; bottom: three modes $k=1, k=2$, and $k=3$ are excited, $\kappa_{N}=0.02, \kappa_{P}=3.2$. Model parameters are $\delta_{N}=0.14, \delta_{P}=0.06, \delta_{\mathbf{v}_{N}}=$ $0.006, \delta_{\mathbf{v}_{P}}=0.004$. Arrows show the current direction of wave movement. 
prey and predators for active directed movement. Increasing the taxis coefficient of one species can compensate lowering the taxis activity of another species. The results obtained are independent of the geometry of the domain $\Omega$ : formulas (3.8) and (3.10) are valid for onedimensional segment, two-dimensional rectangle and circular domain. Taking into account friction forces may change the order of excitation of modes, which otherwise become excited one after another in ascending order. Thus, presence of friction terms in (3.9) makes the model more realistic.

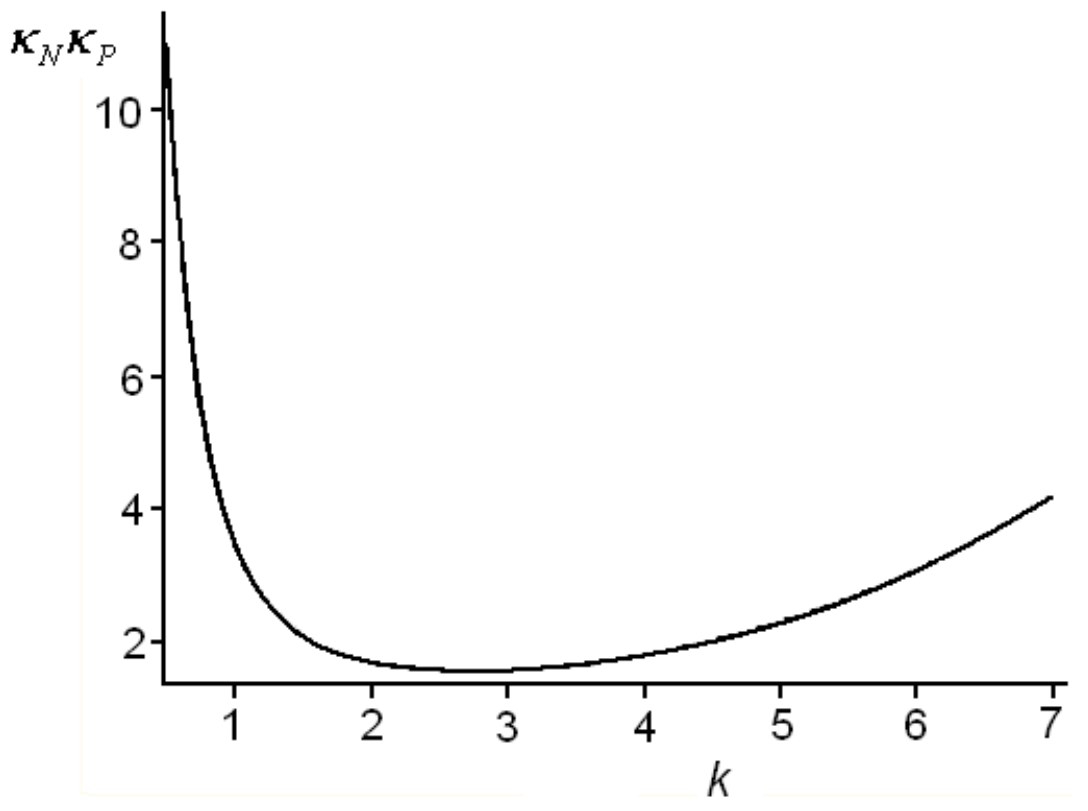

Figure 2: Computed from inequality (3.10) critical curve that shows product of population taxis coefficients vs. the wavenumber of the excited mode. Parameter values are $\delta_{N}=$ $0.02, \delta_{P}=0.002, \delta_{\mathbf{v}_{N}}=0.06, \delta_{\mathbf{v}_{P}}=0.00004, \nu_{N}=2.47$ and $\nu_{P}=1.8$.

We base our model on the hypothesis that taxis acceleration is proportional to the density gradient of another population playing a role of taxis stimulus. This hypothesis allows overcoming known limitations of conventional spatial predator-prey models based on crossdiffusion equations that assume the direct dependence of taxis velocity on density gradient of population-stimulus $[25,7,4,40]$.

Generally speaking, models with cross-diffusion develop the ideas first proposed by Keller and Segel [20] for modelling aggregation of bacteria, applying them to describing waves in excitable two-component systems. Regarding spatial behaviour of predators and prey, the use of prey density in the model as a stimulus for pursuit movement (prey-taxis) of predators and vice versa is just natural. Proposing an alternative to this scheme, we only suggest considering additional substances mediating the indirect pursuit-evasion responses in predator-prey system. Indeed, one can represent system (3.9) in equivalent form as follows:

$$
\frac{\partial N}{\partial t}=\operatorname{div}\left(N \nabla S_{N}\right)+\delta_{N} \Delta N,
$$




$$
\begin{aligned}
\frac{\partial P}{\partial t} & =-\operatorname{div}\left(P \nabla S_{P}\right)+\delta_{P} \Delta P \\
\frac{\partial S_{N}}{\partial t} & =\kappa_{N} P-\nu_{N} S_{N}+\delta_{\mathbf{v}_{N}} \Delta S_{N} \\
\frac{\partial S_{P}}{\partial t} & =\kappa_{P} N-\nu_{P} S_{P}+\delta_{\mathbf{v}_{P}} \Delta S_{P}
\end{aligned}
$$

where $S_{N}$ and $S_{P}$ are potential functions for taxis velocities of prey and predators respectively: $\mathbf{v}_{N}=\nabla S_{N}, \mathbf{v}_{P}=\nabla S_{P}$. According to (4.11) we can interpret $S_{N}$ and $S_{P}$ as a substance like exometabolites (pheromone, odour, etc.) emitted by predators and prey with rates $\kappa_{N}$ and $\kappa_{P}$, respectively. Interestingly, taxis and friction coefficients of system (3.9) became respectively emission and decay rates in (4.11). Stimuli $S_{N}$ and $S_{P}$ decay with rates $\nu_{N}$ and $\nu_{P}$, diffuse with coefficients $\delta_{\mathbf{v}_{N}}$ and $\delta_{\mathbf{v}_{P}}$, respectively repelling and attracting another species. Such substances can exist in the reality but not necessarily: one can consider the potentials $S_{N}$ and $S_{P}$ to be just an abstraction. In form (4.11), equations of our model (3.9) contain the cross-diffusion terms like system (2.4) but unlike (2.4) it easily exhibits complex heterogeneous solutions. Moreover, system (4.11) inherits more traits from the original Keller-Segel [20] chemotaxis model than system (2.4).

Paradoxically, increasing the number of equations in the model by taking into account some stimulus-mediator or the velocity variation greatly simplifies the modelling task because realistic complex heterogeneous dynamics can be obtained in systems with highly simple local kinetics $[15,14,45,2]$ or even without any reaction term in the model as shown in the present study (see also $[36,43]$ ).

The principle difference between systems (3.9) and (2.4) is that two-component model (2.4) assumes that taxis velocities of populations immediately adapts to stimulus distribution while movement of population densities in (3.9) is inertial. The accounting for the inertial delay of taxis response can be achieved either by considering taxis acceleration as in (3.9) or, equivalently, by including additional stimuli mediating pursuit-evasion interaction of prey and predators as in (4.11). What form is more preferable is an open question. The choice should be done in each particular case. On one hand, the latest form seems more convenient if spatial domain $\Omega$ is two- or three-dimensional: the use of scalar potentials $S_{N}$ and $S_{P}$ instead of vectors $\mathbf{v}_{N}$ and $\mathbf{v}_{P}$ reduces the number of equations in the model. On the other hand, identification of a model that explicitly accounts for dynamics of stimulus requires monitoring of their concentrations, the task that can be more difficult than kinematic analysis of animal movements (see, e.g., [28,42]).

Obviously, systems (3.9) and (4.11) present a minimal and thus quite simplified description of complex biological phenomena related to still unexplored behavioural mechanisms working in live systems. The development of the proposed basic scheme shall further approach the reality. In particular, the proposed model does not account for the Weber-Fechner effect, assuming that individuals have an infinite ability to sense the heterogeneity of the rival population. Considering density dependent interrelated diffusion and taxis coefficients similar to those in the classical Keller-Segel chemotaxis model [20] easily removes the problem. However it is important to emphasize that even with simplest constant coefficients 
$\delta_{N}, \delta_{P}, \kappa_{N}$ and $\kappa_{P}$, models (3.9), (4.11) perfectly describe small supercriticality of the bifurcation studied when both perturbations of equilibrium population densities and their gradients remain low.

As a conclusion, we would like to suggest to model massive directional movements of population density of highly organised species (fishes, insects, rodents, etc.) on the basis of the inertial taxis in form (3.9) or (4.11), while spatio-temporal dynamics of micro-organisms whose motion is characterised by low Reynolds number should be modelled by cross-diffusion systems like (2.2) that shall contain nonlinear terms of local kinetics in order to ensure stabilization of dynamic heterogeneous regimes.

Acknowledgements. We thank two anonymous reviewers for their constructive comments. The work was partially supported by the internal grant K-07-T-112 from the Southern Federal University (project "Research-teaching laboratory of Computer Simulations and Modelling of Biological Systems").

\section{References}

[1] R.M. Alexander. Principles of animal locomotion. Princeton University Press, Princeton, 2003.

[2] R. Arditi, Y. Tyutyunov, A. Morgulis, V. Govorukhin, I. Senina. Directed movement of predators and the emergence of density-dependence in predator-prey models. Theoretical Population Biology 59 (2001), 207-221.

[3] F.S. Berezovskaya, G.P. Karev. Bifurcations of running waves in population models with taxis. Uspekhi Fizicheskikh Nauk 9 (1999), 1011-1024 [in Russian].

[4] F.S. Berezovskaya, A.S. Isaev, G.P. Karev, R.G. Khlebopros. The role of taxis in dynamics of forest insects. Doklady Akademii Nauk 365 (1999), 416-419 [in Russian].

[5] S. Camazine, J.-L. Deneubourg, N. Franks, J. Sneyd, G. Théraulaz, E. Bonabeau. Selforganization in biological systems. Princeton University Press, Princeton \& Oxford, 2003.

[6] A. Chakraborty, M. Singh, D. Lucy, P. Ridland. Predator-prey model with prey-taxis and diffusion. Mathematical and Computer Modelling 46 (2007), 482-498.

[7] T. Czárán. Spatiotemporal models of population and community dynamics. Chapman and Hall, London, 1998.

[8] Y.A. Dombrovsky, G.S. Markman. Spatial and temporal ordering in ecological and biochemical systems. Rostov State University, Rostov-on-Don, 1983. [in Russian]

[9] P. Eklöv, S. Diehl. Piscivore efficiency and refuging prey: the importance of predator search mode. Oecologia 98 (1994), 344-353. 
[10] L. Edelstein-Keshet. Mathematical models in biology. McGraw-Hill, New York, 1988.

[11] G. Flierl, D. Grünbaum, S. Levin, D. Olson. From individuals to aggregations: the interplay between behaviour and physics. Journal of Theoretical Biology 196 (1999), 397-454.

[12] L.R. Ginzburg, C.X.J. Jensen. Rules of thumb for judging ecological theories. Trends in Ecology and Evolution 19 (2004), 121-126.

[13] L.R. Ginzburg, M. Colyvan. Ecological orbits: How planets move and populations grow. Oxford University Press, Oxford, 2004.

[14] V.N. Govorukhin, A.B. Morgulis, Y.V. Tyutyunov. Slow taxis in a predator-prey model. Doklady Mathematics 61 (2000), 420-422.

[15] V.N. Govorukhin, A.B. Morgulis, I.N. Senina, Y.V. Tyutyunov. Modelling of active migrations for spatially distributed populations. Surveys of Applied and Industrial Mathematics 6 (1999), 271-295. [in Russian]

[16] D. Grünbaum, A. Okubo. Modelling social animal aggregation. In: Frontiers in mathematical biology (S. Levin, ed.). Springer, New York, 1994, pp. 296-325.

[17] N. Hammerschlag, R.A. Martin, C. Fallows. Effects of environmental conditions on predator-prey interactions between white sharks (Carcharodon carcharias) and Capefurseals (Arctocephaluspusillus pusillus) at Seal Island, South Africa. Environmental Biology of Fishes (2006), DOI 10.1007/s10641-006-9038-z.

[18] A. Huth, C. Wissel. The simulation of fish schools in comparison with experimental data. Ecological Modelling 75/76 (1994), 135-145.

[19] G.R. Ivanitskii, A.B. Medvinskii, M.A. Tsyganov. From disorder to order as applied to the movement of micro-organisms. Soviet Physics Uspekhi 34(4) (1991), 289-315.

[20] E.F. Keller, L.A. Segel. Initiation of slide mold aggregation viewed as an instability. Journal of Theoretical Biology 26 (1970), 399-415.

[21] S.-H. Lee, J.H. Park, T.-S. Chon, H.K. Pak. Prey-flock deformation under a predator atack. Journal of the Korean Physical Society 48 (2006), S236-S240.

[22] B.R. MacKenzie, T. Kiørboe. Encounter rates and swimming behavior of pause-travel and cruise larval fish predators in calm and turbulent laboratory environments. Limnology and Oceanography 40 (1995), 1278-1289.

[23] A.B. Medvinsky, S.V. Petrovskii, I.A. Tikhonova, E. Venturino, H. Malchow. Chaos and regular dynamics in model multi-habitat plankton-fish communities. Journal of Biosciences 26 (2001), 109-120. 
[24] A. Mogilner, L. Edelstein-Keshet. A non-local model for a swarm. Journal of Mathematical Biology 38 (1999), 534-570.

[25] J.D. Murray. Mathematical biology. Springer-Verlag, New York, 1993.

[26] J.D. Murray. Mathematical biology II: Spatial models and biomedical applications. Springer-Verlag, New York, 2003.

[27] A. Okubo, S. Levin. Diffusion and ecological problems: Modern perspectives, SpringerVerlag, New York, 2001.

[28] A. Okubo, H.C. Chiang, C.C. Ebbesmeyer. Acceleration field of individual midges, Anarete pritchardi (Diptera: Cecidomyiidae), within a swarm. Canadian Entomologist 109 (1977), 149-156.

[29] J.K. Parrish, S.V. Viscido, D. Grünbaum. Self-organized fish schools: an examination of emergent properties. Biological Bulletin 202 (2002), 296-305.

[30] S.V. Petrovskii, B.L. Li. Exactly solvable models of biological invasion. CRC Press, Boca Raton, 2006.

[31] S.V. Petrovskii, H. Malchow. A minimal model of pattern formation in a prey-predator system. Mathematical and Computer Modelling 29(8) (1999), 49-63.

[32] T.J. Pitcher, J.K. Parrish. Functions of shoaling behavior in teleosts. In: Behaviour of Teleost Fishes (T.J. Pitcher, ed.). Chapman and Hall, London, 1993, pp. 363-439.

[33] D. Ritz. The benefits of a good school: When it comes to schooling, invertebrates such as shrimps and squids do it like fish, and probably for the same reasons. New Scientist 1761 (1991), 41.

[34] T.C. Roth, S.L. Lima. Use of prey hotspots by an avian predator: purposeful unpredictability? The American Naturalist 169 (2007), 264-273.

[35] N. Sapoukhina, Y. Tyutyunov, R. Arditi. The role of prey-taxis in biological control: a spatial theoretical model. The American Naturalist 162 (2003), 61-76.

[36] I.N. Senina, Y.V. Tyutyunov. Modelling of schooling as a consequence of autotaxis. Zhurnal Obshchei Biologii 63 (2002), 483-488. [in Russian]

[37] Y.M. Svirezhev. Nonlinear waves, dissipative structures and catastrophes in ecology. Nauka, Moscow, 1987. [in Russian]

[38] M.A. Tsyganov, V.N. Biktashev. Half-soliton interaction of population taxis waves in predator-prey systems with pursuit and evasion. Physical Review Letters E 70 (2004), 031901. 
[39] M.A. Tsyganov, V.N. Biktashev, J. Brindley, A.V. Holden, G.R. Ivanitsky. Waves in systems with cross-diffusion as a new class of nonlinear waves. Uspekhi Fizicheskikh Nauk 177(3) (2007), 275-300.

[40] M.A. Tsyganov, J. Brindley, A.V. Holden, V.N. Biktashev. Quasi-soliton interaction of pursuit-evasion waves in a predator-prey system. Physical Review Letters 91 (2003), 218102.

[41] M.A. Tsyganov, J. Brindley, A.V. Holden, V.N. Biktashev. Soliton-like phenomena in one-dimensional cross-diffusion systems: a predator-prey pursuit and evasion example. Physica D 197 (2004), 18-33.

[42] P. Turchin. Quantitative analysis of movement: Measuring and modeling population distribution in animals and plants. Sinauer, Sunderland, 1998.

[43] Y. Tyutyunov, I. Senina, R. Arditi. Clustering due to acceleration in the response to population gradient: a simple self-organization model. The American Naturalist 164 (2004), 722-735.

[44] Y.V. Tyutyunov, N.Y. Sapoukhina, A.B. Morgulis, V.N. Govorukhin. Mathematical model of active migrations as a foraging strategy in trophic communities. Zhurnal Obshchei Biologii 62 (2001), 253-262. [In Russian]

[45] Y.V. Tyutyunov, N.Y. Sapoukhina, I.N. Senina, R. Arditi. Explicit model for searching behaviour of predator. Zhurnal Obshchei Biologii 63 (2002), 137-148. [In Russian]

[46] E. Venturino, A.B. Medvinsky. The role of periodic boundary forcing in plankton pattern formation. Ecological Modelling 140 (2001), 255-270.

[47] J.A. Walker, C.K. Ghalabmor, O.L. Griset, D. McKenney, D.N. Reznick. Do faster starts increase the probability of evading predators? Functional Ecology 19 (2005), 808-815. 